\title{
Medicinal plants traditionally used for the management of female reproductive health dysfunction in Tana River County, Kenya
}

\author{
Catherine Kaluwa Kaingu ${ }^{1, *}$, Jemimah Achieng Oduma ${ }^{1}$, James Mucunu Mbaria ${ }^{2}$, Stephen Gitah Kiama ${ }^{1}$ \\ ${ }^{1}$ Department of Veterinary Anatomy and Physiology, University of Nairobi, P.O Box 30197-00100, Nairobi, Kenya; ${ }^{2}$ Department of \\ Public Health, Pharmacology and Toxicology, University of Nairobi, P.O Box 30197-00100, Nairobi, Kenya
}

\begin{abstract}
Reproductive dysfunction is a major health concern amongst the inhabitants of Tana River County. An ethno botanical study was conducted in Garsen, Itsowe and Ngao sub divisions of Tana River County to document the utilization of medicinal plants for the management of female reproductive ailments. The target population was practicing herbalists from Pokomo, Ormo and Giryama communities in the study area. Structured questionnaires and focussed group discussions were used to collect data. Forty eight plant species distributed in 40 genera and 29 families were documented as being important for the management of pregnancy related complications, menstrual disorders, infertility, fibroids and as contraceptives. The species most frequently cited by the herbalists were fourteen. Fifty two percent of the plant species were probably being mentioned for the first time as being useful in reproductive health management. In conclusion, Tana River has a pool of TMPs with a wealth of indigenous knowledge that needs to be exploited. The plants used to treat dysmenorrhea for example may be important analgesic agents that need further investigation while those with anti-fertility properties may contain steroidal phyto chemical compounds. Such species therefore need further investigation to establish their efficacy and mechanism of action.
\end{abstract}

Keywords medicinal plants, female reproductive ailments, Tana River, Kenya

\section{INTRODUCTION}

Herbal medicines have been used for the treatment of human ailments for thousands of years (Yakubu et al., 2007a; Yakubu and Bukoye, 2009). Recently, there has been renewed interest, spearheaded by World Health Organization (WHO), in the use of medicinal plants by traditional healers in Africa. This interest has led to increased research on traditional medicines.

Traditional medicine as practiced among various African societies is based on the concept that the cause of illness and disease or discomfort is sometimes ascribed to forces arising from angered ancestral spirits or evil spirits and witchcraft. Traditional medicine sees the supernatural as the cause of most major illnesses and factors of one's social and economic environment are all considered in diagnosing physical and mental problems in people's lives. Smaller medical issues however are handled with herbal remedies but even this is holistically applied whereby the whole plant, its physical characteristics like its aroma, taste, color and nutrient value, along with the rituals attending to its preparation and administration are just as important as its pharmacological content (Gessler et al., 1995; Okpako, 1999).

Traditional medicinal practitioners (TMPs) by their nature do not keep records and most of the knowledge they have is passed on verbally from generation to generation (Giday et al., 2010). There is therefore need not just to capture this indigenous knowledge but also to study the plants in order to

*Correspondence: Catherine Kaluwa Kaingu

E-mail: catekaluwa@yahoo.co.uk

Received March 14, 2013; Accepted May 16, 2013; Published May 31,2013

doi: http://dx.doi.org/10.5667/tang.2013.0006

(C)2013 by Association of Humanitas Medicine

TANG / www.e-tang.org provide credible evidence to support therapeutic efficacy claims by herbalists (Sofowora, 1993).

Reproductive issues and ailments constitute $18 \%$ of the global burden of disease for women of reproductive age and are the number one cause of maternal mortality in developing countries (WHO, 2003). Female reproductive ailments range from pregnancy and related complications, fertility issues and menstrual complications. In Tana River County, TMPs are routinely consulted because of their wide indigenous medicinal knowledge base (Swaleh, 1999), a tradition that has persisted in many rural communities due to inequitable health provision.

In Kenya, $75 \%$ of health facilities and personnel are concentrated in urban areas (National Policy of Traditional Medicine, 2005). The national doctor patient ratio is 1: 20,000; but in Tana River County with only 57 health facilities, the doctor: patient ratio is 1: 95,500 emphasizing a serious shortage of both health facilities and staff in the County (Tana River District Strategic Plan, 2005 - 2010). On the other hand, the ratio of TMP to patients is 1: 987 (Kenya Housing and Population report, 2009), suggesting that the TMPs are more readily accessible. In General, health sectors including reproductive health face a number of challenges. According to the Tana River District Strategic Plan (2005 - 2010); issues of major concern in reproductive health sector are; unsafe motherhood, high maternal/child mortality rates and inadequate family planning services.

An ethno botanical survey was carried out in Tana River County to identify and document the plants that are used by traditional herbalists for the management of female reproductive ailments and problems. The plant parts, route of administration, method of preparation, dose and whether the plant was administered as a decoction or concoction was documented.

2013 / Volume 3 / Issue 2 / e17 


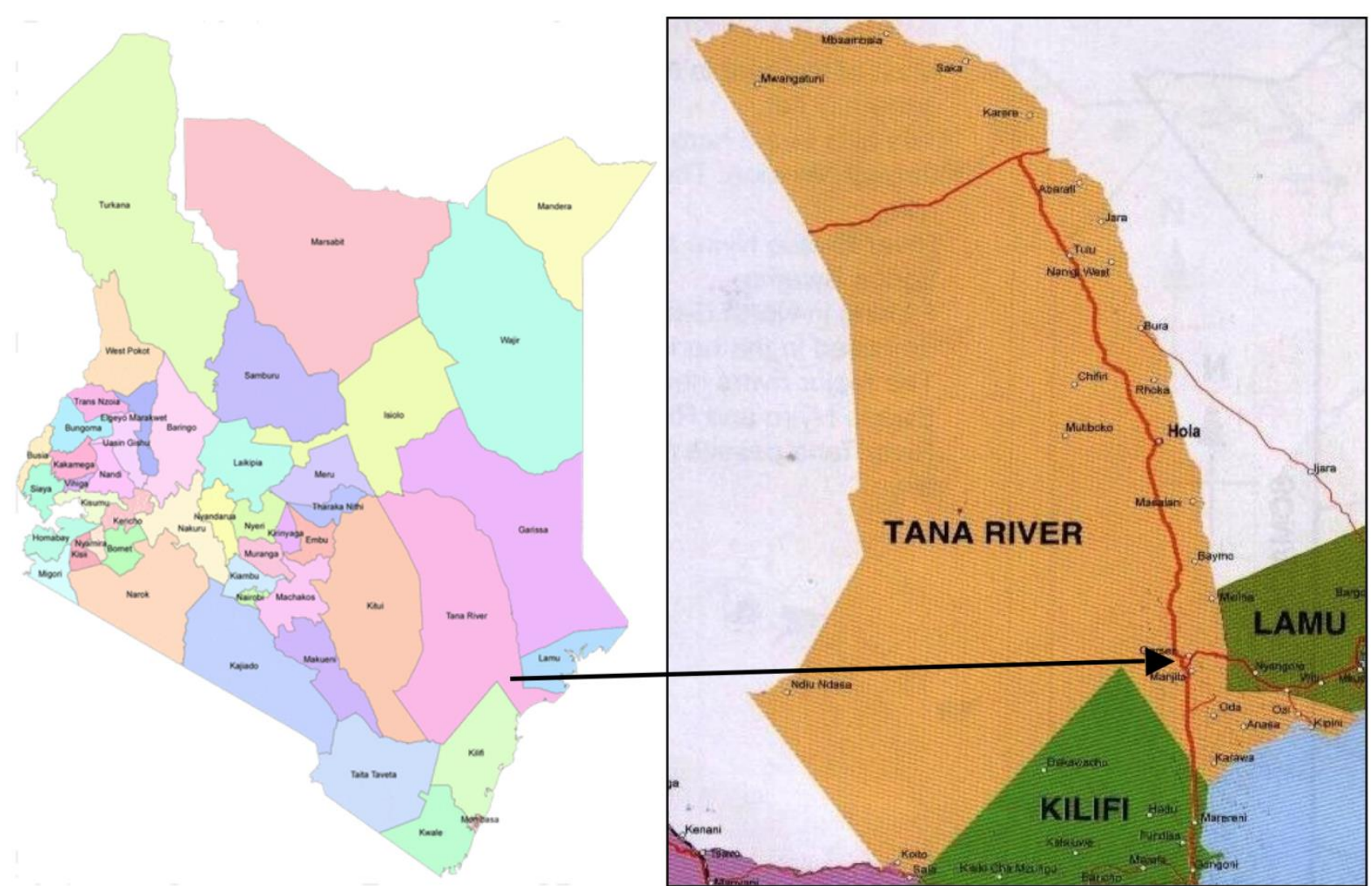

Fig. 1. The map of Tana River County, Kenya (Source: Department of Geography University of Nairobi). Arrow points at area of study: Garsen , Itsowe and Ngao subdivisions.

\section{MATERIAL AND METHODS}

\section{Study area identification and description}

A reconnaissance survey was undertaken in Tana River County in March 2012, to identify key informants for the study. Local administrators were key resource persons in providing information on TMPs. Discussions with these key informants led to Garsen, Itsowe and Ngao subdivisions being chosen as most suitable study areas due to widespread use of herbal medicine and in accessibility to health facilities. The County borders the Indian ocean to the south. It has a coastal strip that is approximately 35 kilometers. The County is divided into 7 administrative divisions, 43 locations and 93 sub locations. It lies between latitude $0^{\circ}$ and $3^{\circ}$ South and longitudes $38^{\circ} 30^{\prime}$ east and $40^{\circ} 15^{\prime}$ east. According to the population and housing census report 2009; the County has a population of 240,075 persons, $72 \%$ of whom live below the poverty line.

\section{Target population, study design and data collection}

The target population for the ethno botanical survey was TMPs and the County has a high number of these. The TMPs were derived from the main tribes living in the study area namely Pokomo, Ormas and Giryama. The University of Nairobi Biosafety, Animal welfare and Ethics Committee reviewed and approved the research protocol. The study design was a cross sectional survey where a systematic random sampling method was used to identify 80 practicing herbalists as participants. Semi structured questionnaires were used to document medicinal plants used by TMPs for the management of reproductive ailments in Garsen, Itsowe and Ngao subdivisions, by a team comprising local translators, botanist and researchers. TMPs were asked to give signed informed consent before participating in the study. The objectives of the study were clearly stated. Quantitative and qualitative data collection methods were applied. Structured questionnaires were administered to the TMPs, and focused group discussions were conducted that allowed for detailed exploration of individual's knowledge and practices about reproductive health ailments and management. The questionnaires were designed to be responsive to the objectives of the study. Interviews with informants were conducted in Pokomo, Orma and Giryama languages assisted by local translators, and responses were recorded in English. A pilot study was conducted earlier to test and re-design the research tools appropriately. The TMPs study variables included age, marital status, education levels, number of years in practice, how they acquired their knowledge and the interventions used to manage reproductive health ailments.

\section{Sample size determination and statistical analysis}

Fisher et al. (1998) formula was used to determine sample size; $\mathrm{n}=\mathrm{Z}^{2} \mathrm{pq} / \mathrm{d}^{2}$ whereby $\mathrm{n}=$ the desired sample size; $\mathrm{z}=$ the standard normal deviate at the required confidence level; $\mathrm{p}=$ the proportion in the target population estimate to have characteristics being measured; $\mathrm{q}=1-\mathrm{p} ; \mathrm{d}=$ the level of statistical significance set. The data was analyzed qualitatively.

\section{Plant identification}

The plants were identified by a taxonomist and voucher specimens deposited at the University of Nairobi Herbarium. The information gathered included vernacular name of plant, species and ailment treated. Plant part, route of administration, method of preparation, dose, duration and whether the remedy was administered as a concoction or decoction was also documented. 


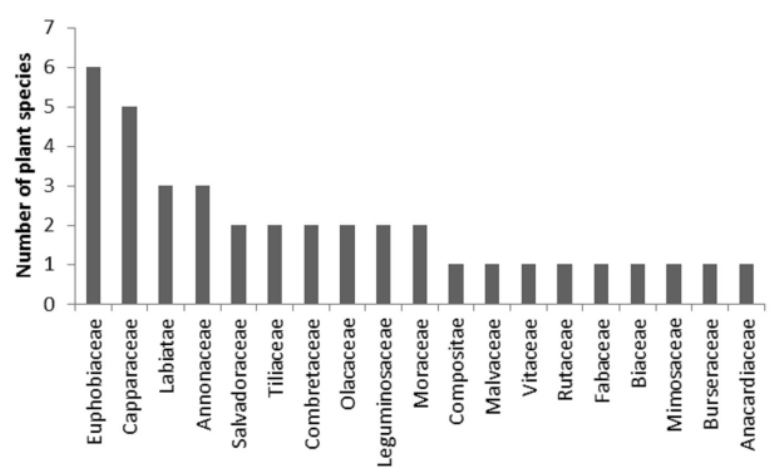

Fig. 2. Commonest plant family documented for the management of female reproductive health dysfunctions in Tana River County.

\section{RESULTS}

A total of 80 herbalists from the three subdivisions were interviewed. The herbalists were mostly elderly people aged 45 years and above and mostly illiterate (53\%), with only 5 having completed primary school and another 2 completed secondary school. Majority of the herbalists $(68 \%)$ were males while ten of the female herbalists practiced also as Traditional Birth Attendants (TBAs). All the herbalists had been in practice for 15 years or more and practically all had acquired their knowledge from relatives. Forty eight medicinal plants were used for the management of reproductive health ailments (Table 1). The plants belonged to 29 families, the commonest based on family use value being Euphorbiaceae, Capparaceae, Labiatae, Annonaceae, Leguminoceae, Tiliaceae, Salvadoraceae, Combretaceae, Olacaceae, Moraceae (Fig. 2). Fig. 3 gives the distribution of reproductive health ailments and the percentage of plants used for their management. A total of 27 plants $(56.3 \%)$ were identified for the management of pregnancy and related problems (Table 1).

Sixteen plants $(32.65 \%)$ were presented for prevention of threatened abortion, 10 plants $(20.8 \%)$ were used to alleviate post-partum hemorrhage, 10 plants $(20.8 \%)$ to manage retained afterbirth, 3 plants $(6.25 \%)$ to alleviate protracted labor, 1 plant

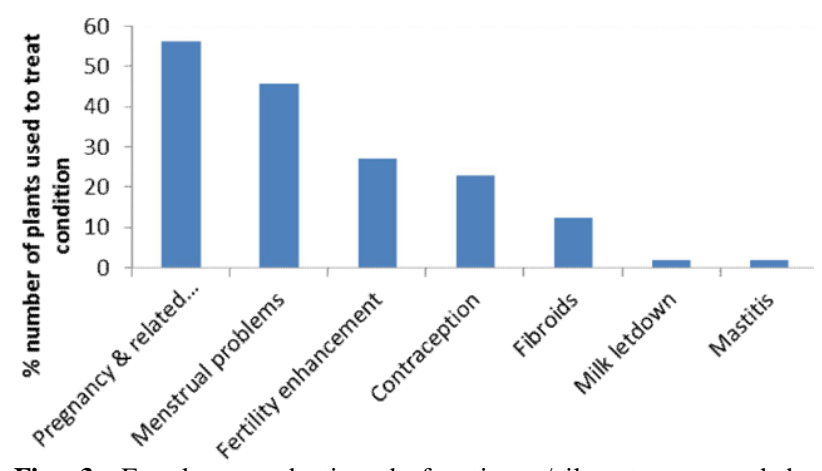

Fig. 3. Female reproductive dysfunctions /ailments managed by herbalists in Tana River County.

(2.08\%) was used to augment or induce labor, 1 plant $(2.08 \%)$ to arrest premature labor, 1 plant $(2.08 \%)$ to manage breach birth and 1 plant $(2.08 \%)$ was used to induce retraction of the uterus after birth. Twenty two $(45.8 \%)$ plants were identified for the management of menstrual disorders; 8 plants $(16.7 \%)$ were presented for the management of menorrhagia, 4 plants $(8.3 \%)$ were used to treat dysmenorrhea, $5(10.42 \%)$ to treat amenorrhea and $5(10.42 \%)$ to manage irregular menses. Thirteen $(27.08 \%)$ plants were used to treat infertility or to enhance fertility. Eleven plants $(22.92 \%)$ were used as contraceptives to suppress fertility after delivery, 6 plants $(12.50 \%)$ were presented for management of fibroids, $1(2.08 \%)$ to induce milk letdown and 1 plant $(2.08 \%)$ ) to treat mastitis.

The data on herbal preparations, mode of administration and part used is presented in Table 1. The most common method of preparation involved boiling or soaking the fresh or dried plant parts in water (decoctions) or ground into powder and taken orally or as infusions. The water extracts were prepared just before consumption or just before steam bath. The most frequent route of administration as reported by the herbalist was oral at $93 \%$, followed by topical application at $7 \%$. Most of the remedies were prepared as concoctions of more than one plant in combination with the principal plant. The most common plant part used was the root $(71 \%)$, followed by the leaf $(22 \%)$, the root bark $(6 \%)$, the stem $(4 \%)$ and the fruit $(2 \%)$.

Table 1. Medicinal plants used to manage female reproductive ailments in Tana River, Kenya

\begin{tabular}{|c|c|c|c|c|}
\hline Family & Plant species, & Local name & Traditional use & $\begin{array}{l}\text { Method of preparation, route of administration } \\
\text { and dose }\end{array}$ \\
\hline Aloeaceae & $\begin{array}{l}\text { Aloe volkensii Engl. } \\
\text { CK027 }\end{array}$ & Hargeis, D'aar (Orma) & Infertility & $\begin{array}{l}\text { Leaves squashed in water. Decoction used to wash } \\
\text { genital area } 3 \text { times daily until effective. }\end{array}$ \\
\hline Anacardiaceae & $\begin{array}{l}\text { Lannea schweinfurthii } \\
\text { (Engl.) Engl. CK001 }\end{array}$ & Mumongoo (Pokomo) & RAB, PPH & $\begin{array}{l}\text { Roots boiled in water and decoction taken orally. } \\
\text { Half glass daily for } 2 \text { days }\end{array}$ \\
\hline Annonaceae & $\begin{array}{l}\text { Uvariodendron kirkii } \\
\text { Verdc. CK008 }\end{array}$ & Msaidizi (Giryama) & Contraceptive & $\begin{array}{l}\text { Root bark boiled in water and decoction taken } \\
\text { orally. One glass daily for } 30 \text { days. Every } 7 \text { days } \\
\text { fresh root bark boiled in water. }\end{array}$ \\
\hline Annonaceae & $\begin{array}{l}\text { Uvaria acuminata oliv } \\
\text { CK023 }\end{array}$ & $\begin{array}{l}\text { Mundagoni, Murori } \\
\text { (Pokomo) }\end{array}$ & $\begin{array}{l}\text { PPH, menorrhagia, } \\
\text { dysmenorrhea }\end{array}$ & $\begin{array}{l}\text { Roots boiled in water and concoction taken orally. } \\
\text { One glass daily for } 5 \text { days. Usually mixed with } \\
\text { Markhamia zanzibarica }\end{array}$ \\
\hline Annonaceae & Uvaria leptocladon & Sholole (Orma) & $\begin{array}{l}\text { Threatened abortion, } \\
\text { infertility, breach birth, } \\
\text { RAB }\end{array}$ & $\begin{array}{l}\text { Roots boiled in water and concoction taken orally. } \\
\text { Half glass } 3 \text { times daily for } 3 \text { days. Mixed with } \\
\text { Croton dichagamus }\end{array}$ \\
\hline Apocynaceae & $\begin{array}{l}\text { Hunteria zaylanica } \\
\text { (zetz.) Gard ex thr var } \\
\text { CK041 }\end{array}$ & $\begin{array}{l}\text { Mutsungutsungu, } \\
\text { (Pokomo) }\end{array}$ & $\begin{array}{l}\text { Induces milk letdown } \\
\text { after delivery }\end{array}$ & Induces milk letdown after delivery \\
\hline Biaceae & $\begin{array}{l}\text { Pergularia daemia } \\
\text { (forsk.) chiov CK046 }\end{array}$ & Mpovu (Pokomo) & RAB & $\begin{array}{l}\text { Roots or leaves boiled in water and decoction } \\
\text { taken orally. One glass daily for } 3 \text { days }\end{array}$ \\
\hline Bignoniaceae & $\begin{array}{l}\text { Markhamia zanzibarica } \\
\text { CK014 }\end{array}$ & Mubwoka (Pokomo) & $\begin{array}{l}\text { Threatened abortion, } \\
\text { infertility, menorrhagia, } \\
\text { dysmenorrhea, } \\
\text { amenorrhea, RAB, } \\
\text { contraceptive, Fibroids }\end{array}$ & $\begin{array}{l}\text { Roots or leaves boiled in water and concoction } \\
\text { taken orally. Half glass twice daily for } 5 \text { days. } \\
\text { Mixed with Salvadora persica and Uvaria } \\
\text { acuminata oliv. }\end{array}$ \\
\hline
\end{tabular}




\begin{tabular}{|c|c|c|c|c|}
\hline Burseraceae & $\begin{array}{l}\text { Commiphora } \\
\text { habessinica }(\mathrm{O} . \mathrm{Berg}) \\
\text { Engl. CK050 }\end{array}$ & Mutsutsu (Pokomo) & $\begin{array}{l}\text { RAB, PPH, post- } \\
\text { partum retraction of } \\
\text { uterus. }\end{array}$ & $\begin{array}{l}\text { Roots boiled in water and decoction taken orally. } \\
\text { Half glass daily for } 4 \text { days }\end{array}$ \\
\hline Capparaceae & $\begin{array}{l}\text { Thylachium thomasii } \\
\text { Gilg CK024 }\end{array}$ & Uhiya, kukube (Orma) & Threatened abortion & $\begin{array}{l}\text { Roots boiled in water and decoction taken orally. } \\
\text { Half glass daily for } 3 \text { days }\end{array}$ \\
\hline Capparaceae & $\begin{array}{l}\text { Boscia coriaceae pax. } \\
\text { CK025 }\end{array}$ & Kalkacha (Orma) & $\begin{array}{l}\text { Threatened abortion, } \\
\text { menorrhagia, } \\
\text { dysmenorrhea, } \\
\text { amenorrhea, irregular } \\
\text { menses, RAB, PPH }\end{array}$ & $\begin{array}{l}\text { Roots boiled in water and concoction taken orally. } \\
\text { Half glass daily for } 5 \text { days. Usually mixed with } \\
\text { Uvaria leptocladon and Combretum hereroense } \\
\text { Schinz }\end{array}$ \\
\hline Capparaceae & $\begin{array}{l}\text { Cadaba ruspolii Gilg } \\
\text { CK032 }\end{array}$ & Ilkavate (Orma) & Threatened abortion & $\begin{array}{l}\text { Roots boiled in water and decoction taken orally. } \\
\text { Half glass daily for } 3 \text { days }\end{array}$ \\
\hline Capparaceae & $\begin{array}{l}\text { Cadaba glandulosa } \\
\text { forsk. CK037 }\end{array}$ & Alakal (Orma) & Infertility & $\begin{array}{l}\text { Roots boiled in water and decoction taken orally. } \\
\text { Half glass daily for } 5 \text { days }\end{array}$ \\
\hline Capparaceae & $\begin{array}{l}\text { Cadaba farinose } \\
\text { CK038 }\end{array}$ & Kumis (Orma) & Infertility & $\begin{array}{l}\text { Roots boiled in water and decoction taken orally. } \\
\text { Half glass daily for } 2 \text { days }\end{array}$ \\
\hline Combretaceae & $\begin{array}{l}\text { Combretum hereroense } \\
\text { Schinz. CK035 }\end{array}$ & Konkon (orma) & $\begin{array}{l}\text { Threatened abortion, } \\
\text { menorrhagia, } \\
\text { dysmenorrhea, } \\
\text { amenorrhea, irregular } \\
\text { menses, RAB, PPH }\end{array}$ & $\begin{array}{l}\text { Roots boiled in water and concoction taken orally. } \\
\text { Half glass } 3 \text { times daily for } 6 \text { days. Mixed with } \\
\text { Uvaria leptacladon roots }\end{array}$ \\
\hline Combretaceae & $\begin{array}{l}\text { Combretum Illairii } \\
\text { Engl. CK049 }\end{array}$ & $\begin{array}{l}\text { Mshinda alume } \\
\text { (Pokomo) }\end{array}$ & $\begin{array}{l}\text { Infertility, PPH, } \\
\text { Contraceptive }\end{array}$ & $\begin{array}{l}\text { Roots boiled in water and decoction taken orally. } \\
\text { Half glass } 2-3 \text { times daily for } 14 \text { days }\end{array}$ \\
\hline Compositae & $\begin{array}{l}\text { Pluchea ovalis (Pers.) } \\
\text { DC CK010 }\end{array}$ & Msasa (Pokomo) & Vaginal Rash & $\begin{array}{l}\text { Leaves boiled in water and decoction used to } \\
\text { wash genitalia for } 1 \text { week }\end{array}$ \\
\hline Euphobiaceae & $\begin{array}{l}\text { Ricinus communis } \mathrm{L} \text {. } \\
\text { CK016 }\end{array}$ & $\begin{array}{l}\text { Mubonye, Mbono } \\
\text { (Pokomo) }\end{array}$ & Contraceptive & $\begin{array}{l}\text { Two dried fruits swallowed daily for } 30 \text { days. The } \\
\text { same dose repeated after } 1 \text { year }\end{array}$ \\
\hline Euphobiaceae & $\begin{array}{l}\text { Acalypha volkensii Pax } \\
\text { CK020 }\end{array}$ & $\begin{array}{l}\text { Mupunga mbuu } \\
\text { (Pokomo) }\end{array}$ & Threatened abortion & $\begin{array}{l}\text { Root bark boiled in water and decoction taken } \\
\text { orally. Half glass daily for } 3 \text { days }\end{array}$ \\
\hline Euphobiaceae & $\begin{array}{l}\text { Croton menyharthii } \\
\text { pax CK021 }\end{array}$ & $\begin{array}{l}\text { Mualikaji, Muyama } \\
\text { (Pokomo) }\end{array}$ & $\begin{array}{l}\text { Contraceptive, PPH, } \\
\text { Threatened abortion, } \\
\text { Infertility, menorrhagia, } \\
\text { Irregular menses }\end{array}$ & $\begin{array}{l}\text { Root and or leaves boiled in water and decoction } \\
\text { taken orally. Half glass } 2-3 \text { times daily for } 5 \text { days }\end{array}$ \\
\hline Euphobiaceae & $\begin{array}{l}\text { Suregada } \\
\text { zanzibariensis Boull. } \\
\text { CK022 }\end{array}$ & $\begin{array}{l}\text { Mudimu tsaka } \\
\text { (Giryama) }\end{array}$ & Contraceptive & $\begin{array}{l}\text { Roots boiled in water and decoction taken orally. } \\
\text { Half glass } 3 \text { times daily for } 4 \text { days }\end{array}$ \\
\hline Euphobiaceae & $\begin{array}{l}\text { Croton dichagamus } \\
\text { CK031 }\end{array}$ & $\begin{array}{l}\text { Qashin a'adha, } \\
\text { Muuqaadhi (Orma) }\end{array}$ & $\begin{array}{l}\text { Threatened abortion, } \\
\text { Infertility }\end{array}$ & $\begin{array}{l}\text { Roots boiled in water and concoction taken orally. } \\
\text { Half glass } 3 \text { times daily for } 6 \text { days. Sometimes } \\
\text { mixed with Uvaria leptocladon roots. }\end{array}$ \\
\hline Euphobiaceae & $\begin{array}{l}\text { Euphorbea uhligiana } \\
\text { pax CK044 }\end{array}$ & Daalid (Orma) & $\begin{array}{l}\text { Threatened abortion, } \\
\text { PPH }\end{array}$ & $\begin{array}{l}\text { Roots boiled in water and decoction taken orally. } \\
\text { Half glass daily for } 2 \text { days }\end{array}$ \\
\hline Fabaceae & $\begin{array}{l}\text { Prosopis juliflora } \\
\text { CK051 }\end{array}$ & Mathenge & $\begin{array}{l}\text { Threatened abortion, } \\
\text { Infertility }\end{array}$ & $\begin{array}{l}\text { Root bark boiled in water and concoction taken } \\
\text { orally. One teaspoonful daily for } 5 \text { days. Mixed } \\
\text { with Zanthoxylum usamel root bark }\end{array}$ \\
\hline Labiatae & $\begin{array}{l}\text { Plectranthus barbatus } \\
\text { Andr. CK015 }\end{array}$ & Papaha (Pokomo) & $\begin{array}{l}\text { Threatened abortion, } \\
\text { RAB, PPH } \\
\text { Contraceptive, } \\
\text { menorrhagia, } \\
\text { amenorrhea, Irregular } \\
\text { menses, Infertility }\end{array}$ & $\begin{array}{l}\text { Roots boiled in water and concoction taken orally. } \\
\text { Half glass daily for } 30 \text { days. Mixed with Cissus } \\
\text { rotundifolia roots for the first } 4 \text { days }\end{array}$ \\
\hline Labiatae & $\begin{array}{l}\text { Ocimum } \\
\text { kilimandscharicum } \\
\text { Gurke CK018 }\end{array}$ & Vumba kuu (Pokomo) & Threatened abortion & $\begin{array}{l}\text { Roots boiled in water and decoction taken orally. } \\
\text { Half glass daily for } 3 \text { days }\end{array}$ \\
\hline Labiatae & $\begin{array}{l}\text { Hoslundia opposite } \\
\text { Vahl CK045 }\end{array}$ & Mtserere & Infertility & $\begin{array}{l}\text { Roots boiled in water and decoction taken orally. } \\
\text { Half glass 2-3 times daily for } 2 \text { days }\end{array}$ \\
\hline Leguminosaceae & $\begin{array}{l}\text { Acacia zanzibarica (S. } \\
\text { Moore) Taub. Var } \\
\text { Zanzibarica CK004 }\end{array}$ & $\begin{array}{l}\text { Muryela (Pokomo), } \\
\text { muhegakululu } \\
\text { (Giryama), Wachu } \\
\text { (Orma) }\end{array}$ & $\begin{array}{l}\text { Irregular menses, } \\
\text { Mastitis }\end{array}$ & $\begin{array}{l}\text { Roots boiled in water and decoction taken orally. } \\
\text { Half glass daily for } 3 \text { days }\end{array}$ \\
\hline Leguminosaceae & $\begin{array}{l}\text { Cassia occidentalis } L \text {. } \\
\text { CK009 }\end{array}$ & Muchoyoko (Pokomo) & RAB, PPH & $\begin{array}{l}\text { Roots or leaves boiled in water and decoction } \\
\text { taken orally. Half glass daily for } 3 \text { days }\end{array}$ \\
\hline Lythraceae & $\begin{array}{l}\text { Lawsonia inermis L. } \\
\text { CK048 }\end{array}$ & Musuruja (Pokomo) & Fibroids & $\begin{array}{l}\text { Roots boiled in water and decoction taken orally. } \\
\text { Half glass daily for } 30 \text { days. After every } 7 \text { days } \\
\text { fresh roots boiled }\end{array}$ \\
\hline Malvaceae & $\begin{array}{l}\text { Thespesia danis Oliv. } \\
\text { CK006 }\end{array}$ & Mudanisa (Pokomo) & Fibroids & $\begin{array}{l}\text { Roots or leaves boiled in water and decoction } \\
\text { taken orally. Half glass daily for } 3 \text { days }\end{array}$ \\
\hline Menispermaceae & $\begin{array}{l}\text { Cissampelos } \\
\text { micronata. A. Rich } \\
\text { CK040 }\end{array}$ & $\begin{array}{l}\text { Chovi, Kivila kya mani } \\
\text { (Pokomo), } \\
\text { Kashikiropaka } \\
\text { (Giryama) }\end{array}$ & $\begin{array}{l}\text { Protracted labor, } \\
\text { Threatened abortion }\end{array}$ & $\begin{array}{l}\text { Roots boiled in water and concoction taken orally. } \\
\text { One glass } 3 \text { times daily for } 4 \text { days. Mixed with } \\
\text { Cassia abbreviate and Strychnos henningsii roots. }\end{array}$ \\
\hline
\end{tabular}




\begin{tabular}{|c|c|c|c|c|}
\hline Mimosaceae & Acacia robusta $\mathrm{CK} 058$ & Munga (Pokomo) & Fibroids & $\begin{array}{l}\text { Roots or leaves boiled in water and concoction } \\
\text { taken orally. One glass } 2 \text { times daily for } 5 \text { days. } \\
\text { Mixed with Cissus rotundifolia roots. }\end{array}$ \\
\hline Moraceae & $\begin{array}{l}\text { Ficus natalensis } \\
\text { Hochst CK013 }\end{array}$ & Mgandi (Pokomo) & Contraceptive & $\begin{array}{l}\text { Roots boiled in water and decoction taken orally. } \\
\text { Half glass daily for } 30 \text { days. After every } 7 \text { days } \\
\text { fresh roots are boiled. }\end{array}$ \\
\hline Moraceae & $\begin{array}{l}\text { Ficus sycomorus L. } \\
\text { CK052 }\end{array}$ & Mukuyu (Pokomo) & $\begin{array}{l}\text { Augment labor, } \\
\text { Protracted labor }\end{array}$ & $\begin{array}{l}\text { Leaves boiled in water and decoction taken orally. } \\
\text { Half glass daily for } 30 \text { days. After every } 7 \text { days } \\
\text { fresh leaves boiled. }\end{array}$ \\
\hline Olacaceae & $\begin{array}{l}\text { Ximenia americana } L \text {. } \\
\text { CK033 }\end{array}$ & $\begin{array}{l}\text { Muntuntuda, } \\
\text { Mtundukula (Pokomo), } \\
\text { Huda hudo (Orma) }\end{array}$ & Contraceptive & $\begin{array}{l}\text { Roots boiled in water and concoction taken orally. } \\
\text { Half glass daily for } 5 \text { days. Mixed with Ochna } \\
\text { holstii roots. }\end{array}$ \\
\hline Olacaceae & $\begin{array}{l}\text { Capparis sepiaria Var. } \\
\text { caffra } \mathrm{CK} 039\end{array}$ & $\begin{array}{l}\text { Hamwalika (Pokomo), } \\
\text { Mugwada paka } \\
\text { (Giryama) }\end{array}$ & Fibroids & $\begin{array}{l}\text { Roots boiled in water and concoction taken orally. } \\
\text { Half glass daily for } 3 \text { days. Sometimes mixed with } \\
\text { Grewia plagiophylla roots }\end{array}$ \\
\hline Passifloraceae & $\begin{array}{l}\text { Adenia gummifera } \\
\text { (Harv.) Harms CK019 }\end{array}$ & Mujoka (Pokomo) & $\begin{array}{l}\text { Menorrhagia, Infertility, } \\
\text { Fibroids }\end{array}$ & $\begin{array}{l}\text { Roots and or stems boiled in water and decoction } \\
\text { taken orally. Half glass daily for } 3 \text { days }\end{array}$ \\
\hline Pedaliaceae & $\begin{array}{l}\text { Pedalium murex } L . \\
\text { CK005 }\end{array}$ & Mbigili (Pokomo) & Protracted labor & $\begin{array}{l}\text { Roots boiled in water and decoction taken orally. } \\
\text { Half glass daily for } 3 \text { days }\end{array}$ \\
\hline Rutaceae & $\begin{array}{l}\text { Citrus sinensis }(\mathrm{L}) \\
\text { Osbeck CK012 }\end{array}$ & Mudimu (Giryama) & Contraceptive, Infertility & $\begin{array}{l}\text { Roots and or stem bark boiled in water and } \\
\text { concoction taken orally. One glass } 3 \text { times daily } \\
\text { for } 3 \text { days. Mixed with Acacia robusta and Cissus } \\
\text { rotundifolia roots. }\end{array}$ \\
\hline Salvadoraceae & $\begin{array}{l}\text { Salvadora persica } \\
\text { L.CK017 }\end{array}$ & $\begin{array}{l}\text { Muswaki, Mujungu } \\
\text { moto (Pokomo) A'adhey } \\
\text { (Orma) }\end{array}$ & Excessive bleeding & $\begin{array}{l}\text { Roots boiled in water and decoction taken orally. } \\
\text { Half glass daily for } 5 \text { days. }\end{array}$ \\
\hline Salvadoraceae & $\begin{array}{l}\text { Dobera glabra (forsk.) } \\
\text { poir CK034 }\end{array}$ & Garas (Orma) & $\mathrm{RAB}$ & $\begin{array}{l}\text { Roots boiled in water and decoction taken orally. } \\
\text { One glass } 2 \text { times daily for } 2 \text { days. }\end{array}$ \\
\hline Sapidaceae & $\begin{array}{l}\text { Allophylus pervilleria } \\
\text { (A.Rich) Engl. CK047 }\end{array}$ & $\begin{array}{l}\text { Mnyanga kitswa } \\
\text { (Pokomo) }\end{array}$ & Infertility & $\begin{array}{l}\text { Roots boiled in water and decoction taken orally. } \\
\text { One glass daily for } 3 \text { days. }\end{array}$ \\
\hline Simorobaceae & $\begin{array}{l}\text { Harrisonia abyssinica } \\
\text { Oliv A. CK042 }\end{array}$ & $\begin{array}{l}\text { Musabini, Muyengwa, } \\
\text { Chewa, (Pokomo) }\end{array}$ & Contraceptive & $\begin{array}{l}\text { Roots boiled in water and concoction taken orally. } \\
\text { One glass } 2-3 \text { times daily for } 3 \text { days. Mixed with } \\
\text { Cassia abbreviate andCissampelos micronata } \\
\text { roots. }\end{array}$ \\
\hline Tiliaceae & $\begin{array}{l}\text { Grewia villosa Willd } \\
\text { CKK } 026\end{array}$ & Ogomdi (orma) & $\begin{array}{l}\text { Threatened abortion, } \\
\text { Contraceptive }\end{array}$ & $\begin{array}{l}\text { Roots boiled in water and decoction taken orally. } \\
\text { Half glass daily for } 30 \text { days. }\end{array}$ \\
\hline Tiliaceae & $\begin{array}{l}\text { Grewia tenax (forssk.) } \\
\text { Fiori. CK028 }\end{array}$ & $\begin{array}{l}\text { Deeka (Orma), } \\
\text { Mubavubavu, Mukawa } \\
\text { wa guba (Pokomo) }\end{array}$ & Infertility, PPH & $\begin{array}{l}\text { Roots boiled in water and concoction taken orally. } \\
\text { Half glass } 3 \text { times daily for } 6 \text { days. Mixed with } \\
\text { Combretum illairii roots. }\end{array}$ \\
\hline Usambarenseceae & $\begin{array}{l}\text { Zanthoxylum usamel } \\
\text { CK011 }\end{array}$ & Safaraji (Pokomo) & Threatened abortion & $\begin{array}{l}\text { Root bark boiled in water and concoction taken } \\
\text { orally. One teaspoonful daily for } 5 \text { days. Mixed } \\
\text { with Prosopis juliflora root bark. }\end{array}$ \\
\hline Vitaceae & $\begin{array}{l}\text { Cissus rotundifolia } \\
\text { (forsk.) CK030 }\end{array}$ & $\begin{array}{l}\text { Mkwembe, Maneke, } \\
\text { Neke (Pokomo), Arma } \\
\text { (Orma) }\end{array}$ & $\begin{array}{l}\text { Threatened abortion } \\
\text { /premature labor, } \\
\text { Contraceptive }\end{array}$ & $\begin{array}{l}\text { Leaves boiled in water and concoction taken } \\
\text { orally. Half glass } 3 \text { times daily for } 4 \text { days. Mixed } \\
\text { with Plectranthus barbatus leaves. }\end{array}$ \\
\hline
\end{tabular}

RAB- Retained after-birth; PPH- Post- partum hemorrhage.

\section{DISCUSSION}

Dried fruits of Ricinus communis were traditionally used in Tana River as contraceptives. Njoroge and Bussmann (2009) reported a similar use in Central province of Kenya. In India, Ramandeep Singh et al. (2011) reported that it had aphrodisiac properties. Euphorbea uhligiana pax was traditionally used for the management of post-partum hemorrhage and prevention of first trimester abortion in Tana River. This corroborates Njoroge and Bussman (2009) who reported a similar use of the plant in Central province of Kenya. Euphorbea candelabrum is used in Loitoktok (Kenya) to treat infertility (Muthee et al., 2011). Plectranthus barbatus is used in Tana River to prevent first trimester abortion. This use contrasts Almeida \& Lemonica (2000) who reported that it was used as an emmenagogue and abortifacient. In Tana River, the species was also used as a contraceptive, menstrual cycle regulator and for management of infertility, post-partum hemorrhage and retained after birth. Ocimum kilimandscharicum Gurke was used to prevent first trimester abortion thereby promoting fertility. This contrasts the study by Gill et al. (2012) who reported on anti-fertility effect of Ocimum sanctum in India. Uvaria acuminate Oliv was used TANG / www.e-tang.org to manage painful menses in Tana River. Ichimaru et al. (2004) reported a similar use in Ethiopia. This probably supports the traditional use of the plant in Tana River. On the other hand; Salvadora persica was used to stop excessive bleeding in Tana River. Darmani et al. (2003) reported its use as a male and female fertility regulator. Grewia hexamita (Ribeiro et al., 2010) was used to regulate the menstrual cycle and promote female fertility. This corroborates the traditional use of Grewia tenax in Tana River for the treatment of infertility. Muthee et al. (2011) reported the use of Ximenia americana for uterine bleeding; Ribeiro et al. (2010) reported its use as a fertility regulator and abortifacient. In Tana River it is used as a contraceptive. Probably its mechanism of action is as an abortifacient. Croton menyharthii pax was the species with the highest use value in Tana River. It was used to treat infertility, prolonged menses, irregular menses, manage post-partum hemorrhage, threatened abortion and as a contraceptive. It was probably being reported for the first time for female reproductive health management as no other study has reported its function in reproduction.

The present study has revealed that traditional medicine practice is not only common in Tana River County of Kenya

2013 / Volume 3 / Issue 2 / e17 
but is socio-culturally acceptable. Traditional healers are known and respected members of the same community in which they practice (Swaleh, 1999). Reproductive health issues that drive women in Tana River County, to visit TMPs are many but similar to those found in other rural parts of Kenya (Kaingu et al., 2011). Several studies have reported that long distances to hospital, unreliable public transport system and lack of financial support are the main constraints that drive people in the rural areas to consult TMPs (Barton and Wamai, 1994; Chuang et al., 2009; Cigand and Laborde, 2003; Kaingu et al., 2011). TMPs are cheap and will rarely deny treatment to patients due to lack of payment. This makes them the most likely to be consulted by the majority rural poor (Kaingu et al., 2011; Kazerooni et al., 2006; Rapkin, 2003).

The present study established that in Tana River County, the custodians of traditional knowledge, including reproductive health knowledge, were all elderly men and women aged over fifty years with long years of practice. Considering that their knowledge was acquired through inheritance from practicing relatives, coupled with the migration of youth to major towns (according to practicing parents and grandparents), there is danger of this knowledge not being passed on to the younger generations for posterity (Kamatenesi-Mugisha and Oryem-Origa, 2005). The lack of documentation is coupled with the lack of systematic conservation to preserve the plants. In this study, the plant part mostly used was the root thereby issues of plant conservation becomes a priority.

Female reproductive ailments managed by traditional healers in Tana River County are shown in Table 1. The commonest ailments were pregnancy and related complications, menstrual problems, infertility and contraception (Fig. 3). The study revealed that pregnant women with signs of threatened abortion readily consulted herbalists and used herbal remedies. Threatened abortion was the most commonly mentioned pregnancy related problem in the community (Table 1). A similar finding was reported by (Kaingu et al., 2011; Chuang et al., 2005 and 2007). Post-partum hemorrhage (PPH) and retained afterbirth $(\mathrm{RAB})$ are the leading cause of maternal mortality and morbidity in developing countries and a concern in developed countries (WHO, 2003, WHO, 2006). Excessive bleeding requires emergency services that would involve administration of uterotonic agents to facilitate the delivery of the placenta (afterbirth). In rural parts of the developing world, such emergency services are non-existent (WHO, 2006). The role played by TMPs in handling PPH and RAB is therefore crucial.

A few plants were reported for the management of delayed and protracted labor and in these cases; TMPs used herbal remedies to induce labor with hardly any hospital referrals. This contrasts similar studies (Kaingu et al., 2011), where some TMPs referred such patients to hospital.

Table 2. Medicinal plants used by herbalists for the management of female reproductive dysfunctions in Tana River County

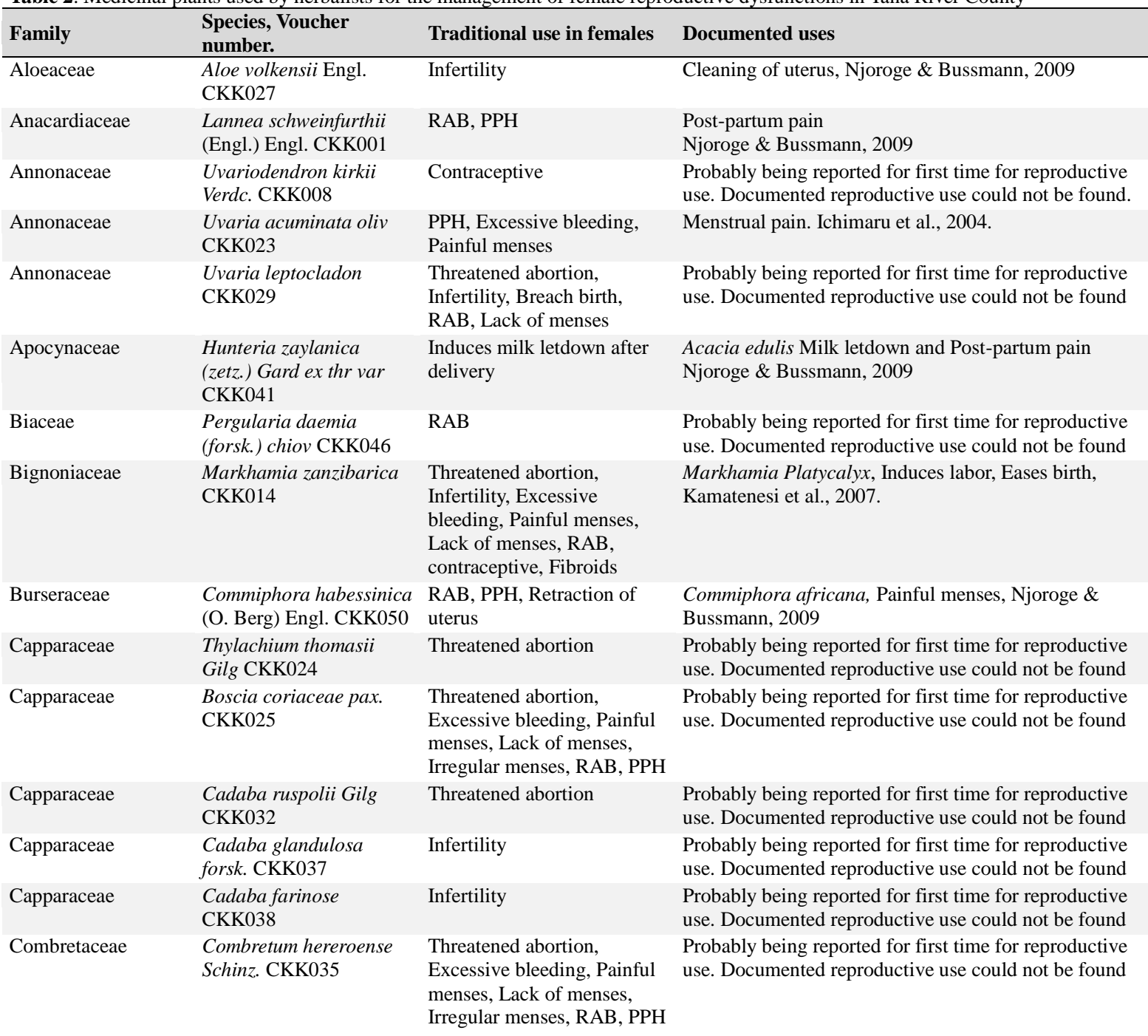

$\mathbf{U V}$

0.14




\begin{tabular}{|c|c|c|}
\hline Combretaceae & $\begin{array}{l}\text { Combretum Illairii Engl. } \\
\text { CKK049 }\end{array}$ & $\begin{array}{l}\text { Infertility, PPH, } \\
\text { Contraceptive }\end{array}$ \\
\hline Compositae & $\begin{array}{l}\text { Pluchea ovalis (Pers.) } \\
\text { DC CKK } 010\end{array}$ & Vaginal Rash \\
\hline Euphobiaceae & $\begin{array}{l}\text { Ricinus communis L. } \\
\text { CKK016 }\end{array}$ & Contraceptive \\
\hline Euphobiaceae & $\begin{array}{l}\text { Acalypha volkensii Pax } \\
\text { CKK020 }\end{array}$ & Threatened abortion \\
\hline Euphobiaceae & $\begin{array}{l}\text { Croton menyharthii pax } \\
\text { CKK } 021\end{array}$ & $\begin{array}{l}\text { Contraceptive, PPH, } \\
\text { Threatened abortion, } \\
\text { Infertility, Prolonged } \\
\text { menses, Irregular menses }\end{array}$ \\
\hline Euphobiaceae & $\begin{array}{l}\text { Suregada zanzibariensis } \\
\text { Boull. CKK022 }\end{array}$ & Contraceptive \\
\hline Euphobiaceae & $\begin{array}{l}\text { Croton dichagamus } \\
\text { CKK031 }\end{array}$ & $\begin{array}{l}\text { Threatened abortion, } \\
\text { Infertility }\end{array}$ \\
\hline Euphobiaceae & $\begin{array}{l}\text { Euphorbea uhligiana pax } \\
\text { CKK044 }\end{array}$ & Threatened abortion, PPH \\
\hline Fabaceae & $\begin{array}{l}\text { Prosopis juliflora } \\
\text { CKK051 }\end{array}$ & $\begin{array}{l}\text { Threatened abortion, } \\
\text { Infertility }\end{array}$ \\
\hline Labiatae & $\begin{array}{l}\text { Plectranthus barbatus } \\
\text { Andr. CKK015 }\end{array}$ & $\begin{array}{l}\text { Threatened abortion, RAB, } \\
\text { PPH Contraceptive, } \\
\text { Excessive bleeding, Lack of } \\
\text { menses, Irregular menses, } \\
\text { Infertility }\end{array}$ \\
\hline Labiatae & $\begin{array}{l}\text { Ocimum } \\
\text { kilimandscharicum } \\
\text { Gurke CKK018 }\end{array}$ & Threatened abortion \\
\hline Labiatae & $\begin{array}{l}\text { Hoslundia opposite Vahl } \\
\text { CKK045 }\end{array}$ & Infertility \\
\hline Leguminosaceae & $\begin{array}{l}\text { Acacia zanzibarica (S. } \\
\text { Moore) Taub. Var } \\
\text { Zanzibarica CKK004 }\end{array}$ & Irregular menses, Mastitis \\
\hline Leguminosaceae & $\begin{array}{l}\text { Cassia occidentalis } L . \\
\text { CKK009 }\end{array}$ & $\mathrm{RAB}, \mathrm{PPH}$ \\
\hline Lythraceae & $\begin{array}{l}\text { Lawsonia inermis L. } \\
\text { CKK048 }\end{array}$ & Fibroids \\
\hline Malvaceae & $\begin{array}{l}\text { Thespesia danis Oliv. } \\
\text { CKK006 }\end{array}$ & Fibroids \\
\hline Menispermaceae & $\begin{array}{l}\text { Cissampelos micronata. } \\
\text { A. Rich CKK } 040\end{array}$ & $\begin{array}{l}\text { Protracted labor, Threatened } \\
\text { abortion }\end{array}$ \\
\hline Mimosaceae & Acacia robusta CKK058 & Fibroids \\
\hline Moraceae & $\begin{array}{l}\text { Ficus natalensis Hochst } \\
\text { CKK013 }\end{array}$ & Contraceptive \\
\hline Moraceae & $\begin{array}{l}\text { Ficus sycomorus L. } \\
\text { CKK } 052\end{array}$ & $\begin{array}{l}\text { Augment labor, Protracted } \\
\text { labor }\end{array}$ \\
\hline Olacaceae & $\begin{array}{l}\text { Ximenia Americana } L . \\
\text { CKK033 }\end{array}$ & Contraceptive \\
\hline Olacaceae & $\begin{array}{l}\text { Capparis sepiaria Var. } \\
\text { caffra CKK039 }\end{array}$ & Fibroids \\
\hline Passifloraceae & $\begin{array}{l}\text { Adenia gummifera } \\
\text { (Harv.) Harms CKK019 }\end{array}$ & $\begin{array}{l}\text { Excessive bleeding, } \\
\text { Infertility, Fibroids }\end{array}$ \\
\hline Pedaliaceae & $\begin{array}{l}\text { Pedalium murex } L . \\
\text { CKK005 }\end{array}$ & Protracted labor \\
\hline Rutaceae & $\begin{array}{l}\text { Citrus sinensis }(\mathrm{L}) \\
\text { Osbeck CKK012 }\end{array}$ & Contraceptive, Infertility \\
\hline Salvadoraceae & $\begin{array}{l}\text { Salvadora persica } \\
\text { L.CKK017 }\end{array}$ & Excessive bleeding \\
\hline Salvadoraceae & $\begin{array}{l}\text { Dobera glabra (forsk.) } \\
\text { poir CKK034 }\end{array}$ & RAB \\
\hline Sapidaceae & $\begin{array}{l}\text { Allophylus pervilleria } \\
\text { (A.Rich) Engl. CKK047 }\end{array}$ & Infertility \\
\hline Simorobaceae & $\begin{array}{l}\text { Harrisonia abyssinica } \\
\text { Oliv A. CKK042 }\end{array}$ & Contraceptive \\
\hline Tiliaceae & $\begin{array}{l}\text { Grewia villosa Willd } \\
\text { CKK026 }\end{array}$ & $\begin{array}{l}\text { Threatened abortion, } \\
\text { Contraceptive }\end{array}$ \\
\hline Tiliaceae & $\begin{array}{l}\text { Grewia tenax (forssk.) } \\
\text { Fiori. CKK028 }\end{array}$ & Infertility, PPH \\
\hline
\end{tabular}

Probably being reported for first time for reproductive use. Documented reproductive use could not be found. Probably being reported for first time for reproductive use. Documented reproductive use could not be found. Antifertility, retained after birth, Njoroge \& Bussmann., 2009

Aphrodisiac, Pallavi et al., 2011

Probably being reported for first time for reproductive use. Documented reproductive use could not be found Probably being reported for first time for reproductive use. Documented reproductive use could not be found

Probably being reported for first time for reproductive use. Documented reproductive use could not be found. Probably being reported for first time for reproductive use. Documented reproductive use could not be found. PPH, Njoroge \& Bussmann., 2009

Probably being reported for first time for reproductive use. Documented reproductive use could not be found.

Emmenagogue, abortifacient Almeida \& Lemonica. 2000; Verissimo LF et al. 2011

Probably being reported for first time for reproductive use. Documented reproductive use could not be found. Probably being reported for first time for reproductive use. Documented reproductive use could not be found.

Infertility, Noumi., 2010

Probably being reported for first time for reproductive use. Documented reproductive use could not be found. Probably being reported for first time for reproductive use. Documented reproductive use could not be found

Probably being reported for first time for reproductive use. Documented reproductive use could not be found Acacia catechu, Acacia nilotica Aphrodisiac, facilitates child birth. Pallavi et al., 2011

F. racemosa aphrodisiac, Pallavi et al., 2011

Female fertility, menstrual cycle, Amri \& Kisangau, 2012. Uterine bleeding, J.K Muthee et al., 2011

Uterine bleeding, J.K. Muthee et al., 2011

Antiabortive, menstrual cycle, women fertility, Ribeiro et al., 2010

Aphrodisiac, Ribeiro et al., 2010

Adenia kirkii, Prolonged menses

Aphrodisiac, Pallavi et al., 2011

Dysmenorrhea, Emmenagogue. Suryawanshi, 2011

Male and female fertility Darmani et al., 2003

Probably being reported for first time for reproductive use. Documented reproductive use could not be found. Probably being reported for first time for reproductive use. Documented reproductive use could not be found. Aphrodisiac, Pallavi et al., 2011

Probably being reported for first time for reproductive use. Documented reproductive use could not be found Grewia hexamita menstrual cycle, women fertility, post-delivery cleaning of uterus Ribeiro et al., 2010 


$\begin{array}{llll}\text { Usambarenseceae } & \begin{array}{l}\text { Zanthoxylum usamel } \\ \text { CKK011 }\end{array} & \text { Threatened abortion } & \text { Post-partum weakness, Njoroge \& Bussmann., 2009 } \\ \text { Vitaceae } & \begin{array}{l}\text { Cissus rotundifolia } \\ \text { (forsk.) CKK030 }\end{array} & \begin{array}{l}\text { Threatened abortion } \\ \text { /Premature labor, } \\ \text { Contraceptive }\end{array} & \begin{array}{l}\text { Probably being reported for first time for reproductive } \\ \text { use. Documented reproductive use could not be found. }\end{array} \\ \end{array}$

RAB- Retained after-birth; PPH- Post- partum hemorrhage.

Menstrual disorders were the second most mentioned ailments in this study. Literature indicates that numerous effects including physical, hormonal and emotional disorders can disrupt the normal menstrual cycle resulting in complications such as absence or abnormal cessation of menstruation (amenorrhea), heavy menstrual bleeding, (menorrhagia), and dysmenorrhea (severe painful menses) (McEvoy et al., 2004; Meduri and Touraine, 2003; Rapkin, 2003). Herbal remedies have proven effective in relieving the pain and discomfort of menstrual disorders. In the present study, menstrual problems were managed by 22 plants (45.8\%) suggesting a high prevalence of such ailments. Similar high prevalence of menstrual disorders has also been reported in other studies (Yassin, 2012). The most common menstrual complaints in this study were menorrhagia, irregular menses, amenorrhea, and dysmenorrhea respectively. This agrees in part with previous studies conducted in Israel (Goldestein et al., 2006), Turkey (Talatu and Egbunu, 2007), England (Houston et al., 2006) and Egypt (Yassin, 2012) where dysmenorrhea and premenstrual syndrome (PMS) were the most prevalent menstrual complaints.

Infertility was the third commonest problem in this study. WHO estimates that approximately 8 - 10\% (50 - 80 million people worldwide) of couples experiences some form of infertility problems whose prevalence varies from region to region (Nagendra and Jayachandra, 2010). Many women consulted herbalists in order to enhance their fertility. The treatment for infertility by use of herbs is worldwide (Deka and Kalita, 2011). However, no individual herb is considered especially useful for promoting fertility. In Africa, India and China for instance, a lot of plants have been used in various combinations to treat infertility (Deka and Kalita, 2011; Ugwah-Oguejiofor et al., 2011; Nagendra and Jayachandra, 2010.

In the present study, although 13 plants were presented for the treatment of infertility or to enhance fertility, 6 plants were also presented for treatment of fibroids, a condition that is linked not only to painful menses and excessive bleeding but also to infertility. The herbalists seemed to clearly recognize the presence of fibroids and claimed to not only control their growth but also shrink the large growths.

Herbal practitioners in this study presented eleven plants which they claimed were used to suppress fertility by preventing conception rather than as abortifacients. The importance of plants as a source of antifertility (contraceptive) drugs has been investigated by many researchers for years (Farnsworth et al., 1975; Yakubu et al., 2007a. Yakubu et al., 2007b) and availability of such plants with anti-fertility properties would be of great benefit in developing countries because such drugs would be easily available and affordable (Goonasekera et al., 1995). The minimal uptake of modern contraceptive methods among rural communities in sub-Saharan Africa is generally due to lack of access to orthodox medicine and contraceptive options in particular (Adebisi and Bello, 2011). In Tana River County the presence of a district hospital at Ngao that offers family planning services did not prevent women from consulting herbalists and instead emphasized the dependence of rural communities on traditional remedies perhaps due to safety considerations.

\section{CONCLUSION}

Reproductive dysfunction is a major obstacle to social-economic development amongst the inhabitants of Tana River County. The area has a pool of TMPs with a wealth of indigenous knowledge that needs to be exploited. The plants used to treat dysmenorrhea for example may be important analgesic agents that need further investigation while others reported for instance as anti-fertility herbs may contain steroidal phyto chemical compounds. Such species therefore need further investigation to establish their active phyto chemical compounds and bio-activity.

\section{ACKNOWLEDGEMENTS}

The authors acknowledge financial support from Regional Initiative in Science Education - African Natural product Network (RISE-AFNNET) that is funded by the Carnegie Cooperation. The funding enabled the ethno botanical study to be carried out successfully. Technical support in identification of plant specimen by Mr. Patrick Chalo Mutiso of Nairobi university herbarium is acknowledged. Authors also thank the Pokomo, Orma and Giryama TMPs for their invaluable information during the study. The authors have no conflicting financial interest.

\section{CONFLICT OF INTEREST}

The authors declare that there was no conflict of interest.

\section{REFERENCES}

Almeida FCG, Lemonica IP. The toxic effect of Coleus barbatus B. on the different periods of pregnancy in rats. J Ethnopharmacol. 2000;73:53-60.

Amri E, Kisangau DP. Ethnomedicinal study of plants used in villages around Kimboza forest reserve in Morogoro, Tanzania. J Ethnobiol Ethnomed. 2012;8:1-7.

Barton TG, Wamai, G. Equity and Vulnerability: A Situation Analysis of Women, Adolescents and Children in Uganda. (Republic of Uganda: National Council Report for Children), 1994.

Chuang CH, Lai JN, Wang JD, Chang PJ, Chen PC. Prevalence and related factors of Chinese herbal medicine use in pregnant women of Taipei 1985-1987. Taiwan J Public Health. 2005;24:335-347.

Chuang CH, Hsieh WS, Guo YL, Lin SH, Lin SJ, Chen P. Chinese herbal medicines used in pregnancy: a population-based survey in Taiwan. Pharmacoepidemiol Drug Saf. 2007;16:464-468.

Chuang CH, Chang PJ, Hsieh WS, Tsai YJ, Lin SJ, Chen PC. 
Chinese herbal medicine use in Taiwan during pregnancy and the post partum period: a population-based cohort study. Int $\mathbf{J}$ Nurs Stud. 2009;46:787-795.

Cigand C, Laborde A. A herbal infusion used for induced abortion. J Toxicol Clin Toxicol. 2003;41:235-239.

Darmani H, Al-Hiyasat AS, Elbetieha AM, Alkofahi A. The effect of an extract of Salvadora persica (meswak, chewing stick) on fertility of male and female mice. Phytomedicine. 2003;10:63-65.

Deka M, Kalita JC. Preliminary phytochemical analysis and acute oral toxicity study of Clitoria ternatea Linn. roots in Albino mice. IRJP. 2011;2:139-140.

Farnsworth NR, Bingel AS, Cordell GA, Crane FA, Fong HHS. Potential value of plants as source of new antifertility agents II. J Pharm Sci. 1975;64:717-754.

Fisher AA, Lang JE, Stoeckel JE, Townsend JW. Handbook for Family Planning Operations and Research Design. $2^{\text {nd }}$ ed. (Republic of Kenya: Population Council of Nairobi), 1998.

Gessler MC, Msuya DE, Nkunya MHH, Schar A, Heinrich M, Tanner M. Traditional healers in Tanzania: sociocultural profile and three short portraits. J Ethnopharmacol. 1995;48:145-160.

Giday M, Asfaw Z, Woldu Z. Medicinal plants of Melnit ethnic group of Ethiopia: an ethnobotanical study. J Ethnopharmacol. 2010;124:513-521.

Gill D, Soni N, Sagar B, Raheja S, Agrawal S. Ocimum kilimandscharicum: A systematic review. J Drug Deliv Ther. 2012;2:45-52.

Goldestein F, Ferber A, Grantot M. The Association between somatization and perceived ability: Roles in dysmenorrhea among Israel Arab adolescents. Psychosom Med. 2006;68:136-142.

Goonasekera MM, Gunawardana VK, Jayasena K, Mohammed SG, Balasubramaniam S. Pregnancy terminating effect of Jatropha curcas in rats. J Ethnopharmacol. 1995;47:117-123.

Houston A, Abraham A, Huang Z,'Angelo L. Knowledge, attitudes and consequences of menstrual health in urban adolescent females. J Pediatr Adolesc Gynecol. 2006;19:271-275.

Ichimaru M, Nakatani N, Takahashi T, Nishiyama Y, Moriyasu M, Kato A, Mathenge SG, Juma FD, Nganga JN. Cytotoxic C-Benzylated Dihydrochalcones from Uvaria acuminate. Chem Pharm Bull (Tokyo). 2004;52:138-141.

Kaingu CK, Oduma JA, Kanui TI. Practices of Traditional Birth Attendants in Machakos District, Kenya. J Ethnopharmacol. 2011;137:495-502.

Kamatenesi-Mugisha M, Oryem-Origa H. Traditional herbal remedies used in the management of sexual impotence and erectile dysfunction in western Uganda. Afr Health Sci. 2005;5:40-49.

Kamatenesi-Mugisha M, Oryem-Origa H. Medicinal plants used to induce labour during childbirth in Western Uganda. J Ethnopharmacol. 2007;109:1-9.
Kazerooni T, Mousavizadeh K, Abdollahee A, Sarkarian M, Sattar A. Abortifacient effects of Prangos ferulacia on pregnant rats. Contraception. 2006;73:554-556.

McEvoy M, Chang J, Coupey SM. Common menstrual disorders in Adolescence: Nursing interventions. MCN Am J Matern Child Nurs. 2004;29:41-49.

Meduri GP, Touraine IB. Delayed Puberty and Primary Amenorrhea Associated with a Novel Mutation of the Human Follicle-Stimulating Hormone Receptor: Clinical, Histological, and Molecular Studies. J Clin Endocrin Metab. 2003;88:3491-3498.

Muthee JK, Gakuya DW, Mbaria JM, Kareru PG, Mulei CM, Njonge FK. Ethnobotanical study of anthelmintic and other medicinal plants traditionaly used in Loitoktok district of Kenya. J Ethnopharmacol. 2011;135:15-21.

Nagendra J, Jayachandra S. Treating infertility by Chinese herbs. J Postgrad Med Inst. 2010;24:336-338.

Njoroge GN, Bussmann RW. Ethnohterapeutic management of Sexually Transmitted Diseases (STDs) and reproductive health conditions in Central Province of Kenya. IJTK. 2009;8:255-261.

Noumi E. Treating fibromyoma with herbal medicines in South Cameroon. IJTK. 2010;9:736-741.

Okpako, David T., Traditional African medicine: Theory and pharmacology explored. Trends Pharmacol Sci. 1999;20:482-486.

Pallavi KJ, Ramandeep S, Sarabjeet S, Karam S, Mamta F, Vinod S. Aphrodisiac agents from medicinal plants: A Review. J Chem Pharm Res. 2011;3:911-921.

Rapkin A. A Review of Treatment of Premenstrual Syndrome and Premenstrual Dysphoric Disorder. Psychoneuroendocrinol ogy. 2003;28:39-53.

Ribeiro A, Romeiras MM, Tavares J, Faria MT. Ethnobotanical survey in Canhane village, district of Massingir, Mozambique: Medicinal plants and traditional knowledge. J Ethnobiol Ethnomed. 2010;6:33.

Sofowora A. Recent trends in research into African medicinal plants. J Ethnopharmcol. 1993;38:209-214.

Suryawanshi JAS. An overview of Citrus aurantium used in treatment of various diseases. African $J$ Plant Science. 2011;5:390-395.

Swaleh A: Ethnoveterinary Medicine in Ormaland-Kenya. (UK Master's thesis in Tropical Animal production and Health, Edinburgh University), 1999.

Talatu S, Egbunu J. Menstrual experiences of adolescents in a secondary school. J. Turkish-German Gynecol Assoc. $2007 ; 8: 7-16$

Yakubu MT, Akanji MA, Oladiji AT. Evaluation of anti-androgenic potentials of acqueous extract of chromolaena aduratum (L) K.R. leaves in male rats. Andrologia. 2007a;39:235-243 
Medicinal plants for female reproductive health management

Yakubu MT, Oladiji AT, Akanji MA. Evaluation of Biochemical indices of male rat reproductive function and testicular histology in wistar rats following chronic administration of aqueous extract of Fadogia agrestis (Schweinf. Ex Heirn) stem. AJBR. 2007b;1:156-163.
Yakubu MT, Bukoye BB. Abortifacient potentials of the aqueous extract of Bambusa vulgaris leaves in pregnant Dutch rabbits. Contraception. 2009;80:308-313.

Yassin SAT. Herbal remedy used by rural adolescent girls with menstrual disorders. J Am Sci. 2012;8:467-473. 